\title{
A New Method of Sensorless Vector Control for Induction Motors
}

\section{Using Instantaneous Reactive Power}

$\begin{array}{lll}\text { Member } & \text { Mineo Tsuji } & \text { (Nagasaki University) } \\ \text { Student-member } & \text { Shuo Chen } & \text { (Nagasaki University) } \\ \text { Member } & \text { Katsuhiro Izumi } & \text { (Nagasaki University) } \\ \text { Member } & \text { Eiji Yamada } & \text { (Nagasaki University) }\end{array}$

\begin{abstract}
This paper proposes a new method of sensorless vector control for induction motors, in which the motor speed is estimated by both instantaneous reactive power and variation of magnetizing current. The proposed system is constructed in a synchronously rotating reference frame and is very simple. Another advantage is that the proposed system can work in regenerating mode without any special technique. A linear model is deduced to analyze system stability. According to the trajectories of poles and zeroes, the system gain is selected to be suited for operating in a wide speed range and in motoring or regenerating mode. The feasibility of the proposed system is verified by digital simulation.
\end{abstract}

Keywords: induction motor, sensorless vector control, instantaneous reactive power, speed estimation, stability analysis

\section{Introduction}

Induction motors have been predominantly the most widely used motors in industrial and domes. tic applications for reasons of simplicity, cost, robustness and reliability. Speed sensor used in the most effective vector-controlled schemes does present problems. It lowers the system reliability, especially in hostile environments, and requires careful cabling arrangement with special attention to electrical noise ${ }^{(1)}$. In addition, a speed sensor is difficult to be mounted on motor shift for some special purpose. To overcome these problems, a large variety of different schemes without speed sensor has been reported ${ }^{(2) \sim(4)}$. Based on stator terminal measurements, the rotor speed can be estimated by an equivalent circuit model of the induction motor. The estimated speed accuracy and the system stability are greatly under the influence of the model parameters, especially stator and rotor resistance ${ }^{(5)}$. In addition, speed sensorless control schemes increase software intricacy more or less and require high-performance processors. However, sensorless vector control is necessary to reduce system complication and to increase reliability for applicability ${ }^{(4)}$.

To overcome the above problems, an approach of indirect vector control to estimate motor speed by instantaneous reactive power is proposed ${ }^{(6)}$. An advantage of this approach is that the speed estimation is robust against the stator resistance variation. Another advantage is achieving much wider-bandwidth speed control. However, a disadvantage is that this approach is unstable in regenerating mode ${ }^{(7)}$. To solve this problem, an approach with the Extended Kalman Filter algorithm is proposed ${ }^{(7)}$. However, this approach is complicated. Stability analysis of the whole system is difficult because the above two approaches are constructed in a stationary reference frame.

In this paper, we propose a new method and study the system stability ${ }^{(8)}$. The motor speed is estimated by both instantaneous reactive power and variation of magnetizing current. This instantaneous reactive power is obtained from the vector control theory ${ }^{(9)}$. Therefore, the new system is simpler than Peng's one. Another advantage is that this system can do its work even in 
regenerating mode without any complicated technique. Because the new system is constructed in a synchronously rotating reference frame, a linear model of the whole system is easy to be deduced. Then, the influence of this system gain is fully taken into consideration in different operating conditions (at low or high speed, in motoring or regenerating mode). According to the analytical investigation, various kinds of control gains are selected to be suited for the system different operations. The feasibility of the proposed system is verified by digital simulation.

\section{Description of Proposed System}

An induction motor can be described by voltage and current models in a synchronously rotating reference frame as follows:

$$
\begin{aligned}
& e_{s d}=\left(r_{s}+\sigma L_{s} p\right) i_{s d}-\omega^{*} \sigma L_{s} i_{s q}+e_{m d} \\
& e_{s q}=\omega^{*} \sigma L_{s} i_{s d}+\left(r_{s}+\sigma L_{s} p\right) i_{s q}+e_{m q} \\
& 0=-\sigma_{r} M i_{s d}+\sigma_{r} \psi_{r d}+\omega_{r} \psi_{r q}+\frac{L_{r}}{M} e_{m d} \\
& 0=-\sigma_{r} M i_{s q}-\omega_{r} \psi_{r d}+\sigma_{r} \psi_{r q}+\frac{L_{r}}{M} e_{m q}
\end{aligned}
$$

where, $i_{s d}, i_{s q}: d-q$ axis stator current;

$$
e_{s d}, e_{s q}: d-q \text { axis stator voltage; }
$$$$
\psi_{r d}, \psi_{r q}: d-q \text { axis flux linkage; }
$$

$\omega_{r}:$ rotor angular speed;

$\omega^{*}$ : electrical angular speed;

$p$ : differential operator;

$$
\sigma_{r}=r_{r} / L_{r}, \sigma=1-M^{2} /\left(L_{s} L_{r}\right) \text {. }
$$

And the other symbols used in the above equations are shown in Table 1. The counter EMF $e_{m d}$ and $e_{m q}$ can be expressed as

$$
\begin{aligned}
& e_{m d}=\frac{M}{L_{r}}\left(p \psi_{r d}-\omega^{*} \psi_{r q}\right) \\
& e_{m q}=\frac{M}{L_{r}}\left(p \psi_{r q}+\omega^{*} \psi_{r d}\right)
\end{aligned}
$$

According to the vector control theory, a synchronously rotating reference frame is chosen for $\psi_{r q}=0$. The instantaneous reactive power into the rotor is defined as

$$
q_{m}=i_{s d} e_{m q}-i_{s q} e_{m d}
$$

The actual currents $i_{s d}$ and $i_{s q}$ can be replaced with $i_{s d}^{*}$ and $i_{s q}^{*}$ if the current control is ideal.
Table 1. Parameter of 3-phase IM

\begin{tabular}{lcl}
\hline \multicolumn{1}{c}{ Parameter } & Symbol & Value \\
\hline Rated output power & & $2.2 \mathrm{~kW}$ \\
Rated torque & & $12.2 \mathrm{~N} \cdot \mathrm{m}$ \\
Rated Current & & $\mathbf{8 . 6 \mathrm { A }}$ \\
Rated rotational speed & & $1720 \mathrm{rpm}$ \\
Moment of Inertia & $J$ & $0.0671 \mathrm{~kg} \cdot \mathrm{m}^{2}$ \\
Pole number & $P$ & 4 \\
Stator resistance & $r_{s}$ & $\mathbf{0 . 0 6 6 2} \Omega$ \\
Rotor resistance & $r_{r}$ & $\mathbf{0 . 0 6 4 5 \Omega}$ \\
Stator self inductance & $L_{s}$ & $\mathbf{0 . 0 8 6 \mathrm { H }}$ \\
Rotor self inductance & $L_{r}$ & $\mathbf{0 . 0 8 6 ~ H}$ \\
Magnetizing inductance & $M$ & $\mathbf{0 . 0 8 2 ~ H}$ \\
\hline
\end{tabular}

From eq.(1) and eq.(2), the instantaneous reactive power of the voltage model used in the controller can be written as

$$
\begin{gathered}
q_{m}=i_{s d}^{*}\left\{e_{s q}-\omega^{*} \sigma L_{s} i_{s d}^{*}-\left(r_{s}^{*}+\sigma L_{s} p\right) i_{s q}^{*}\right\} \\
-i_{s q}^{*}\left\{e_{s d}-r_{s}^{*} i_{s d}^{*}+\omega^{*} \sigma L_{s} i_{s q}^{*}\right\}
\end{gathered}
$$

where $i_{s d}^{*}$ is constant. From eq.(3), the $d$-axis flux in the controller is constant and can be written as

$$
\psi_{r d}^{*}=M i_{s d}^{*}
$$

According to the above description, the counter EMF of the current model can be expressed as

$$
\begin{aligned}
& \hat{e}_{m d}=0 \\
& \hat{e}_{m q}=\frac{M^{2}}{L_{r}} \omega^{*} i_{s d}^{*}
\end{aligned}
$$

Therefore, the instantaneous reactive power of the current model can be written as

$$
\hat{q}_{m}=\frac{M^{2}}{L_{r}} \omega^{*} i_{s d}^{*}
$$

From eq.(4), the flux speed can be written as

$$
\omega^{*}=\hat{\omega}_{r}+\sigma_{r}^{*} \frac{i_{s q}^{*}}{i_{s d}^{*}}
$$

Here $\hat{\omega}_{r}$ is the estimated speed in a sensorless vector control system. We propose a new method to estimate rotor speed as follows:

$$
\begin{gathered}
q_{m}-\hat{q}_{m}=e_{s q} i_{s d}^{*}-e_{s d} i_{s q}^{*}-\omega^{*} L_{s}\left(i_{s d}^{*}+\sigma i_{s q}^{*}\right) \\
-\sigma L_{s} i_{s d}^{*} p i_{s q}^{*} \\
\hat{\omega}_{r}=K_{\omega p}\left(1+\frac{1}{T_{\omega i} p}\right)\left(q_{m}-\hat{q}_{m}\right) \\
+K_{e}\left(1+\frac{1}{T_{e} p}\right)\left(i_{s d}^{*}-i_{s d}\right)
\end{gathered}
$$

As the speed command changes greatly, the motor operating state changes between motoring mode and regenerating mode temporarily. It 
causes variations in the $d$-axis actual current and the $d$-axis rotor flux. To decrease the error between the actual speed and the estimated speed, the error of the $d$-axis current is added to estimate the rotor speed. In addition, the speed estimation is robust against stator resistance because eq.(14) does not contain stator resistance. In order to avoid the differentiation of the $q$-axis current in eq.(14), $p i_{s q}^{*}$ is approximated by a new variable $p q$ as

$$
\begin{aligned}
& q \equiv \frac{1}{1+T_{q} p} i_{s q}^{*} \\
& p i_{s q}^{*} \approx p q=\frac{i_{s q}^{*}}{T_{q}}-\frac{q}{T_{q}}
\end{aligned}
$$

Fig.1 shows the proposed system. The speed PI control is expressed as

$$
i_{s q}^{*}=K_{s}\left(1+\frac{1}{T_{s} p}\right)\left(\omega_{r}^{*}-\hat{\omega}_{r}\right)
$$

The current PI control is written as

$$
\begin{aligned}
& e_{s d}^{*}=K_{p}\left(1+\frac{1}{T_{i} p}\right)\left(i_{s d}^{*}-i_{s d}\right)-\omega^{*} \sigma L_{s} i_{s q}^{*} \\
& e_{s q}^{*}=K_{p}\left(1+\frac{1}{T_{i} p}\right)\left(i_{s q}^{*}-i_{s q}\right)+\omega^{*} L_{s} i_{s d}^{*}
\end{aligned}
$$

\section{Stability Analysis}

The analysis of the proposed system shown in Fig. 1 is presented. To simplify the stability analysis, the motor voltages are assumed to be controlled ideally, or more precisely,

$$
e_{s a}=e_{s a}^{*}, \quad e_{s b}=e_{s b}^{*}, \quad e_{s c}=e_{s c}^{*}
$$

To derive the equation for IM in the $d-q$ axis revolving synchronously with $\omega^{*}$, eq.(21) can be rewritten as

$$
e_{s d}=e_{s d}^{*}, \quad e_{s q}=e_{s q}^{*}
$$

\subsection{Steady-State Solution}

The steady-state solution of the proposed system can be obtained by setting $p=0$. The estimated rotor speed is set equal to the speed command. Load torque $T_{L}, i_{s d}^{*}$ and $\omega_{r}^{*}$ can be given arbitrarily. For convenience of analysis, a given $T_{L}$ is replaced by a given slip frequency. From eq.(3) and eq.(4), the fluxes of IM can be calculated as follows:

$$
\begin{aligned}
& \psi_{r d}=\frac{\sigma_{r} M\left[\sigma_{r} i_{s d}^{*}+\left(\omega^{*}-\omega_{r}\right) i_{s q}^{*}\right]}{\sigma_{r}^{2}+\left(\omega^{*}-\omega_{r}\right)^{2}} \\
& \psi_{r q}=\frac{\sigma_{r} M\left[\sigma_{r} i_{s q}^{*}-\left(\omega^{*}-\omega_{r}\right) i_{s d}^{*}\right]}{\sigma_{r}^{2}+\left(\omega^{*}-\omega_{r}\right)^{2}}
\end{aligned}
$$

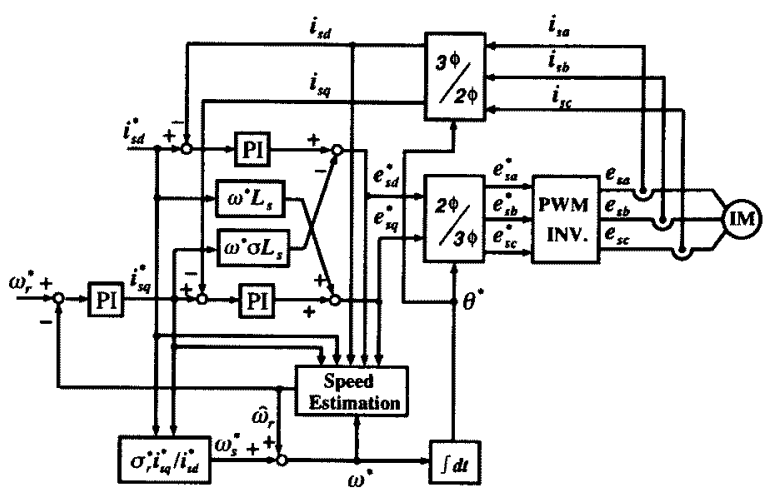

Fig.1. Block diagram of the proposed system

The stator voltages can be calculated from eq.(1) and eq.(2) as follows:

$$
\begin{aligned}
& e_{s d}=r_{s} i_{s d}^{*}-\omega^{*} \sigma L_{s} i_{s q}-\frac{M}{L_{r}} \omega^{*} \psi_{r q} \\
& e_{s q}=\omega^{*} \sigma L_{s} i_{s d}^{*}+r_{s} i_{s q}+\frac{M}{L_{r}} \omega^{*} \psi_{r d}
\end{aligned}
$$

Therefore, $i_{s q}^{*}$ can be determined by the following equation.

$$
e_{s q} i_{s d}^{*}-e_{s d} i_{s q}^{*}-\omega^{*} L_{s}\left(i_{s d}^{*}+\sigma i_{s q}^{* 2}\right)=0
$$

\subsection{Linear Model}

The system stability is analyzed by a linear model that is deduced from the above nonlinear equations at a steady-state operating point. The linear model of IM can be derived from eq.(1) to eq.(4) and the equation of motion.

$$
p \Delta \boldsymbol{x}_{s}=\boldsymbol{A}_{s} \Delta \boldsymbol{x}_{s}+\boldsymbol{B}_{s} \Delta \boldsymbol{u}_{s}+\boldsymbol{B}_{L} \Delta T_{L}
$$

where, $\Delta \boldsymbol{x}_{s}=\left[\begin{array}{llllll}\Delta i_{s d} & \Delta i_{s q} & \Delta \psi_{r d} & \Delta \psi_{r q} & \Delta \omega_{r}\end{array}\right]^{T}$

$$
\Delta u_{s}=\left[\begin{array}{lll}
\Delta e_{s d} & \Delta e_{s q} & \Delta \omega^{*}
\end{array}\right]^{T}
$$

The linear model of the controller is expressed as

$$
\begin{aligned}
p \Delta z & =\boldsymbol{A}_{x} \Delta \boldsymbol{x}_{s}+\boldsymbol{A}_{z} \Delta z_{z}+\boldsymbol{B}_{z} \Delta \boldsymbol{u}_{s}+\boldsymbol{B}_{r} \Delta \omega_{r}^{*} \\
\Delta \boldsymbol{u}_{s} & =\boldsymbol{F}_{x} \Delta \boldsymbol{x}_{s}+\boldsymbol{F}_{z} \Delta z+\boldsymbol{F}_{r} \Delta \omega_{r}^{*}
\end{aligned}
$$

where, $\Delta z=\left[\begin{array}{llllll}\Delta e_{\omega} & \Delta e_{s} & \Delta e_{v d} & \Delta e_{v q} & \Delta q & \Delta \hat{\omega}_{r}\end{array}\right]^{T}$ The state variables $\Delta e_{\omega}, \Delta e_{s}, \Delta e_{v d}$ and $\Delta e_{v q}$ are caused by PI controllers for the speed estimation, speed control, $d \cdot q$ axis current control, respectively. In order to deduce the state equation, a first order lag element is added to calculate $\hat{\omega}_{r}$ in eq.(15). The above matrixes are given in Appendix. From eq.(28), eq.(29) and eq.(30), the linear model of the whole system can be written as

$$
p \Delta \boldsymbol{x}=\boldsymbol{A} \Delta \boldsymbol{x}+\boldsymbol{B} \Delta \omega_{r}^{*}+\boldsymbol{B}_{L}^{\prime} \Delta T_{L}
$$

where, $\Delta x=\left[\begin{array}{ll}\Delta x_{s}^{T} & \Delta z^{T}\end{array}\right]^{T}$ 


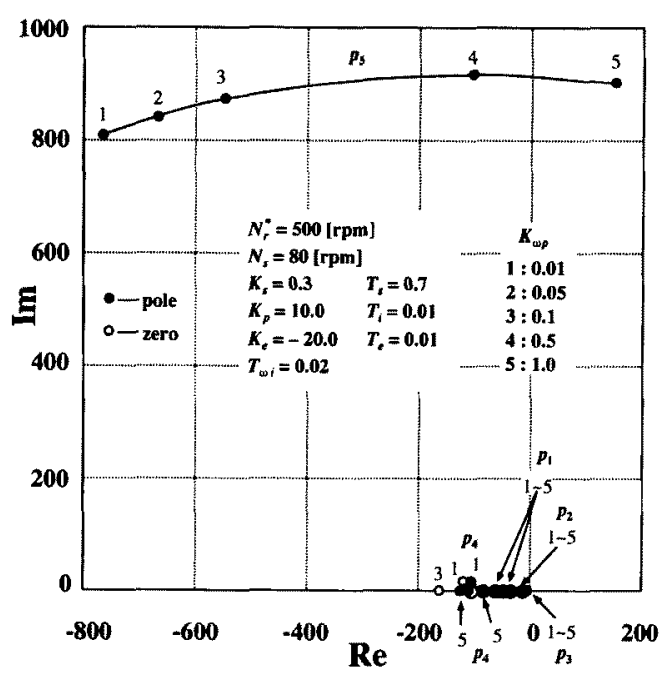

(a) $K_{\omega p}$

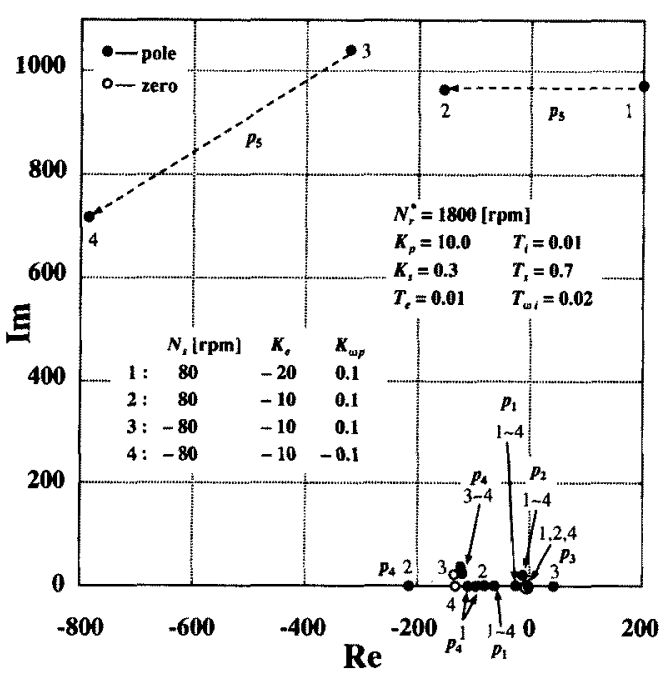

(c) $N_{r}=1800[\mathrm{rpm}]$

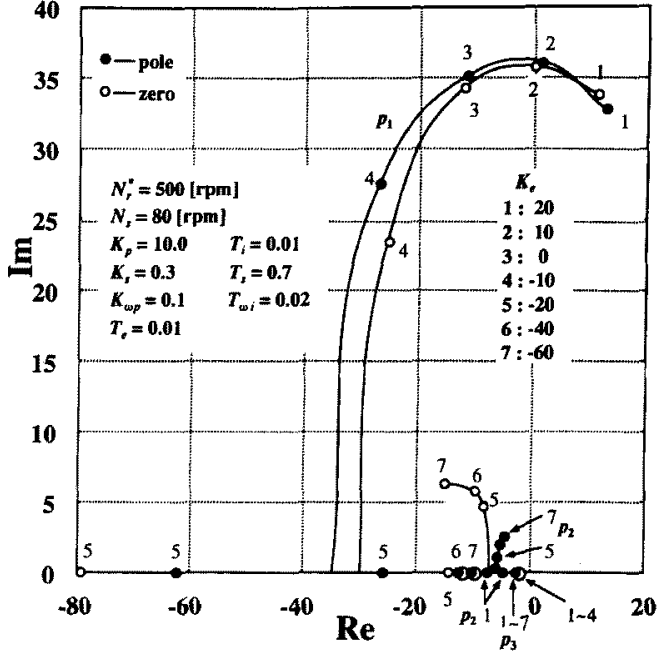

(b) $K_{e}$

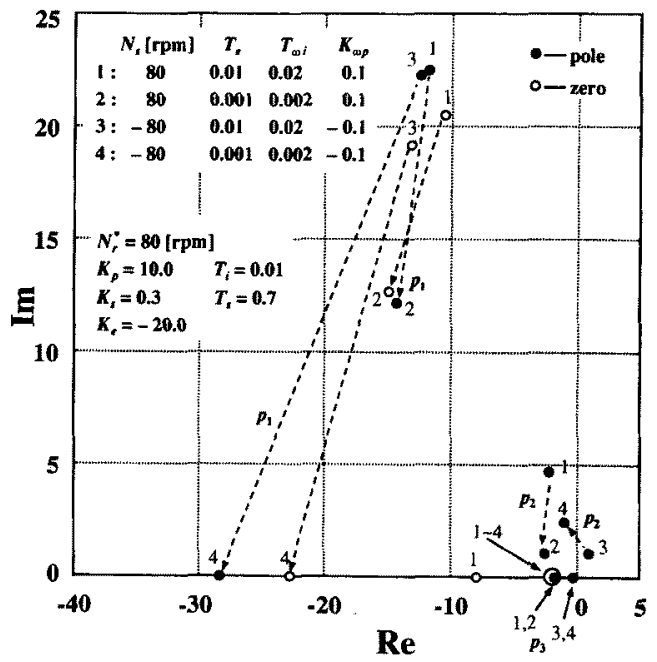

(d) $N_{r}=80[\mathrm{rpm}]$

Fig. 2. Root trajectories of poles and zeros

$$
\begin{gathered}
\boldsymbol{A}=\left[\begin{array}{cc}
\boldsymbol{A}_{s}+\boldsymbol{B}_{s} \boldsymbol{F}_{x} & \boldsymbol{B}_{s} \boldsymbol{F}_{z} \\
\boldsymbol{A}_{x}+\boldsymbol{B}_{z} \boldsymbol{F}_{x} & \boldsymbol{A}_{z}+\boldsymbol{B}_{z} \boldsymbol{F}_{z}
\end{array}\right] \\
\boldsymbol{B}=\left[\begin{array}{c}
\boldsymbol{B}_{s} \boldsymbol{F}_{r} \\
\boldsymbol{B}_{z} \boldsymbol{F}_{r}+\boldsymbol{B}_{r}
\end{array}\right], \quad \boldsymbol{B}_{L}^{\prime}=\left[\begin{array}{c}
\boldsymbol{B}_{L} \\
\mathbf{0}
\end{array}\right]
\end{gathered}
$$

The speed command is chosen as the input variable and the actual speed is chosen as the output variable as follows:

$$
\Delta \omega_{r}=C \Delta x
$$

So, the speed transfer function can be given as

$$
G(s)=\frac{\Delta \omega_{r}}{\Delta \omega_{r}^{*}}=\frac{\operatorname{Cadj}(s \boldsymbol{I}-\boldsymbol{A}) \boldsymbol{B}}{|s \boldsymbol{I}-\boldsymbol{A}|}
$$

\subsection{Results of the Analysis}

In the following study, the parameters of 3phase induction motor given in Table 1 are used.
Fig.2 (a) shows the root trajectories of poles and zeros on the s-plane calculated by eq.(33), in which $K_{\omega p}$ changes from 0.01 to 1 . When $K_{\omega p}$ increases, the pole $p_{5}$ is located in an unstable region and the system becomes unstable, as No. 5 of $p_{5}$ in Fig.2 (a). A suitable value of $K_{\omega p}$ is selected to be 0.1 . Fig.2 (b) shows the root trajectories in which $K_{e}$ changes from -60 to 20 . The pole $p_{1}$ moves into an unstable region when $K_{e}$ is a positive. So, the value of $K_{e}$ must be smaller than zero. Fig.2 (c) shows the root trajectories, in which motor speed is $1800 \mathrm{rpm}$ and slip frequency is 80 rpm or $-80 \mathrm{rpm}$ (full load). The pole $p_{5}$ moves into a stable region when $K_{e}$ changes from -20 to -10 , as No. 1 and No. 2 of $p_{5}$. If $K_{\omega p}$ is a negative, another pole moves into a stable region in regenerating mode, as No. 3 and No. 4 of $p_{3}$. From this 


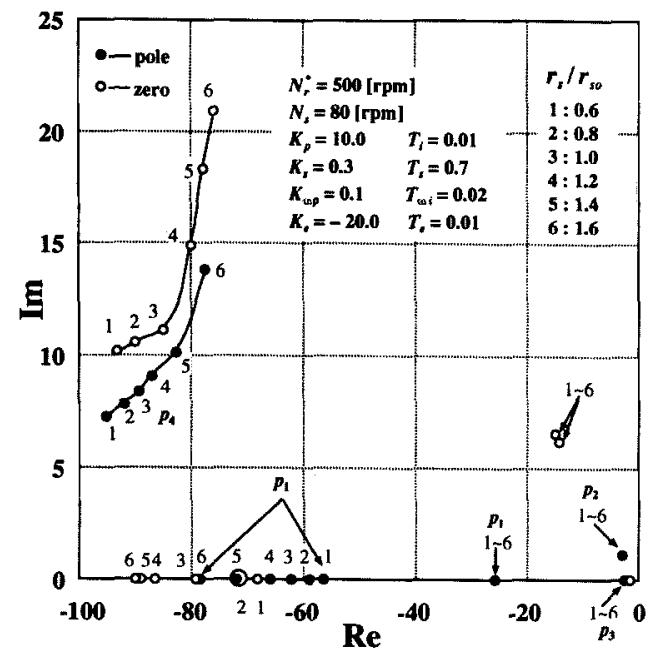

(a) $\boldsymbol{r}_{\mathrm{s}}$

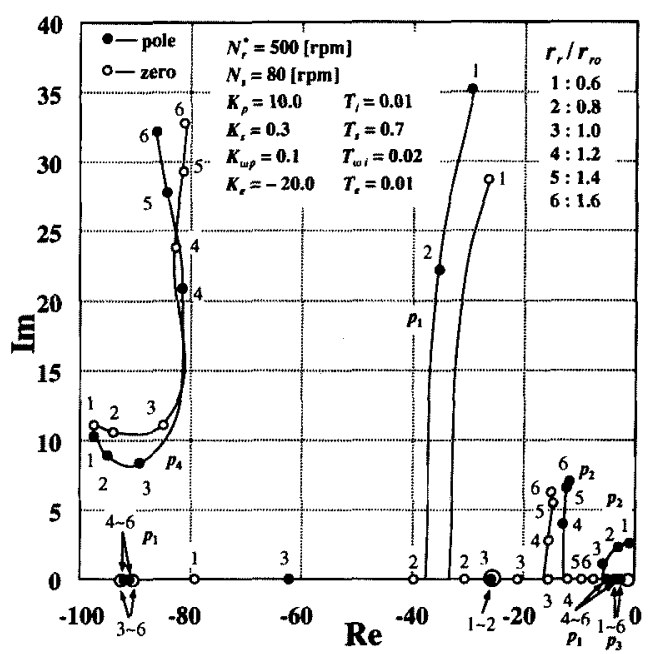

(b) $\boldsymbol{r}_{r}$

Fig.3. Trajectories of poles and zeros with variation of stator or rotor resistance

figure, we know that a $K_{e}$ of -10 is suited to operate at higher speed and a $K_{\omega p}$ of -0.1 is suited to operate in regenerating mode.

Fig.2 (d) shows the root trajectories, in which motor speed is $80 \mathrm{rpm}$ and slip frequency is -80 $\mathrm{rpm}$ or $80 \mathrm{rpm}$. From this figure, we know that the system is more stable if $T_{e}$ changes from 0.01 to 0.001 and $T_{\omega i}$ changes form 0.02 to 0.002 . If the motor is operating in regenerating mode and a $K_{\omega p}$ is -0.1 , No. 3 of the pole $p_{2}$ moves into a stable region, as No. 4 of this pole. As a conclusion, a set of adaptive system gain shown in Table 2 is selected for operating in a wide speed range and in motoring or regenerating mode.

Fig.3 (a) shows the root trajectories, in which the actual value of stator resistance $r_{s}$ changes from $0.6 r_{s o}$ to $1.6 r_{s o}, r_{s o}$ is the nominal value of stator resistance. The stator resistance $r_{s}$ has no influence over the system stability because $r_{s}$ is not used for speed estimation. Fig. 3 (b) shows the root trajectories in which the actual value of rotor resistance $r_{r}$ changes from $0.6 r_{r o}$ to $1.6 r_{r o}$, and $r_{r o}$ is the nominal value of rotor resistance. The $p_{2}$ shown in this figure will move into an unstable region when $r_{r}$ is smaller than $0.6 r_{r o}$. However, this case can be avoided in practice.

Fig.4 shows the operation area in which the system gain shown in Table 2 is used for calculation and rotor resistance changes form $0.8 r_{r o}$ to $1.5 r_{r o}$. Although there is an unstable area in lower-speed range when the slip frequency is a negative, the proposed system can work in a wide speed range, as shown in this figure.
Table 2. Adaptive PI gain for this system

\begin{tabular}{|c|c|c|c|c|c|}
\hline \multirow{2}{*}{$\begin{array}{c}\hat{N}_{r} \\
{[r p m]}\end{array}$} & \multirow{2}{*}{$\boldsymbol{K}_{\boldsymbol{e}}$} & \multirow{2}{*}{$\boldsymbol{T}_{\varepsilon}$} & \multirow{2}{*}{$\boldsymbol{T}_{\omega i}$} & \multicolumn{2}{|c|}{$\boldsymbol{K}_{\omega p}$} \\
\hline & & & & $i_{s q}^{*} \geq 0$ & $i_{s q}^{*}<0$ \\
\hline \multirow{3}{*}{$\begin{array}{c}1800 \\
? \\
800 \\
? \\
500 \\
? \\
200 \\
? \\
0\end{array}$} & -10.0 & & \multirow[b]{2}{*}{0.02} & \multirow{3}{*}{0.1} & \multirow{3}{*}{-0.1} \\
\hline & \multirow[b]{2}{*}{-20.0} & & & & \\
\hline & & 0.001 & 0.002 & & \\
\hline
\end{tabular}

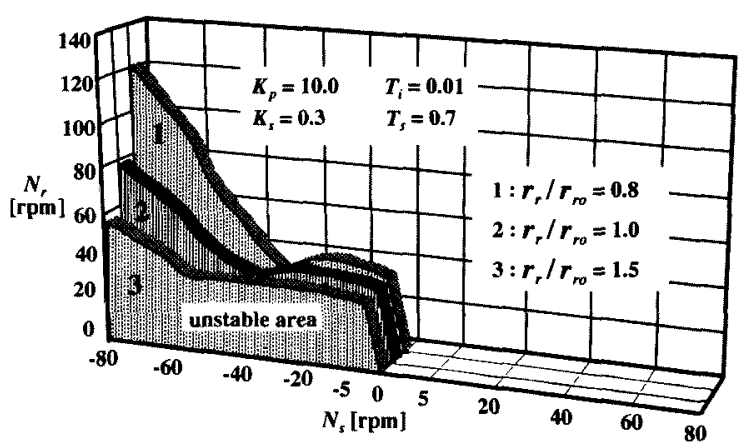

Fig.4. Unstable area with $r_{r}$

\section{Digital Simulation}

To study the performance of the system during transients, the most common approach is to use the differential equations of IM model. For verifying the proposed system, the nonlinear model is used for simulation. Variables of the motor part are still in a continuous system and calculated by 
the Runge-Kutta method. The control part is digitized by the backward Euler method and the sampling period $T_{c}$ is $256 \mu \mathrm{s}$. The dead time caused by the digitized controller is equal to $T_{c}$ and considered in the following simulation. For example, eq.(15) can be rewritten as follows:

$$
\begin{aligned}
\hat{\omega}_{r}{ }^{k} & =\hat{\omega}_{r}{ }^{k-1}+K_{a p}\left(1+\frac{T_{c}}{T_{a i}}\right)\left(q_{m}{ }^{k-1}-\hat{q}_{m}{ }^{k-1}\right) \\
& -K_{a p}\left(q_{m}{ }^{k-2}-\hat{q}_{m}{ }^{k-2}\right)-K_{e}\left(i_{s d}{ }^{*}-i_{s d}{ }^{k-2}\right) \\
& +K_{e}\left(1+\frac{T_{c}}{T_{e}}\right)\left(i_{s d}^{*}-i_{s d}{ }^{k-1}\right)
\end{aligned}
$$

Fig.5 shows the transient responses for the step change of speed command. Fig.5 (a) shows the simulated result that $K_{e}$ is zero and the step change of speed command is $200 \mathrm{rpm}$. When the speed command steps down to $500 \mathrm{rpm}$ at 6 second, the actual speed can not converge to $500 \mathrm{rpm}$. Although the motor is still desired to work in motoring mode, the torque-producing current is a negative. This is because only the information of instantaneous reactive power is not enough to control an induction motor in regenerating mode. This result is similar to that reported by Kubota et $a I^{(7)}$. To overcome this problem, both of the information of instantaneous reactive power and the variation of magnetizing current are used to estimate motor speed. Fig.5 (b) shows the result that $K_{e}$ is -20 and the slip frequency is $80 \mathrm{rpm}$. Fig.5 (c) shows the result that the slip frequency is $-80 \mathrm{rpm}$. From these figures, it is confident that the proposed method can work in both motoring and regenerating mode.

As stator resistance increases with the inner temperature of IM, the actual value of $r_{s}$ is greater than the nominal value when the motor is in operation. In the following simulations, the actual value of $r_{s}$ is set to be $1.5 r_{s o}$.

Fig.6 shows the result that the motor operates at lower speed and the step change of speed command is $40 \mathrm{rpm}$. Fig. 6 (a) is the result that the slip frequency is $80 \mathrm{rpm}$. Fig. 6 (b) is the result that the slip frequency is $-30 \mathrm{rpm}$. Fig. 7 shows the result that the motor operates at higher speed and the step change of speed command is $200 \mathrm{rpm}$. Fig.7 (a) is the result that the motor works in motoring mode. Fig. 7 (b) is the result that the motor works in regenerating mode. From Fig.6 and Fig. 7 , we can know that the proposed system can

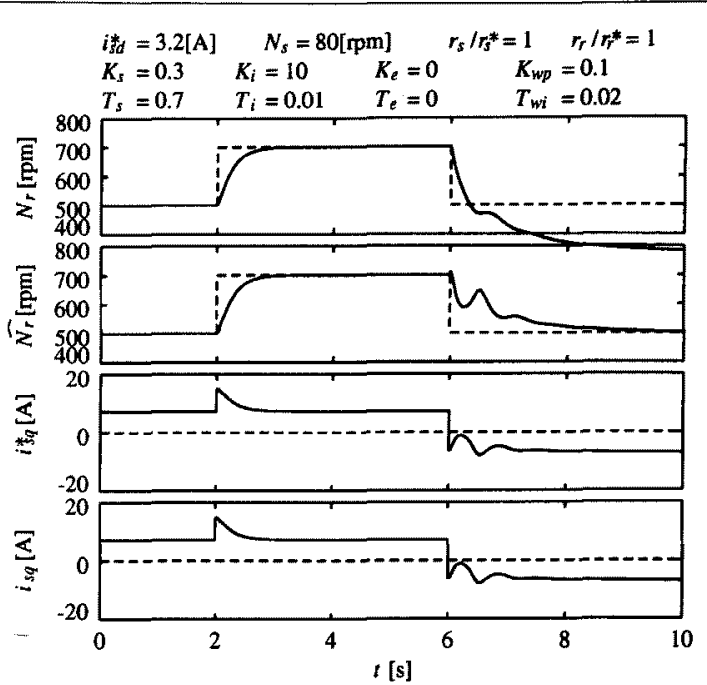

(a) $K_{e}=0$

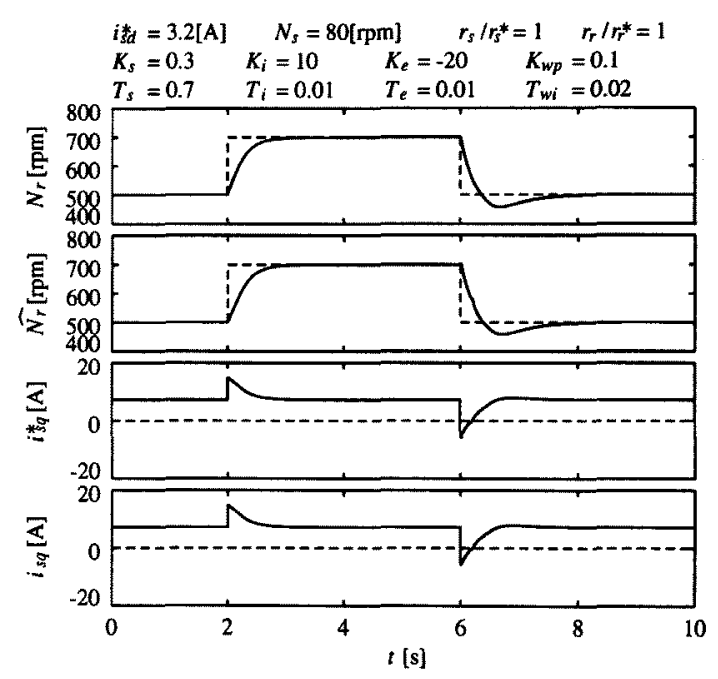

(b) Motoring mode with $K_{e}=-20$

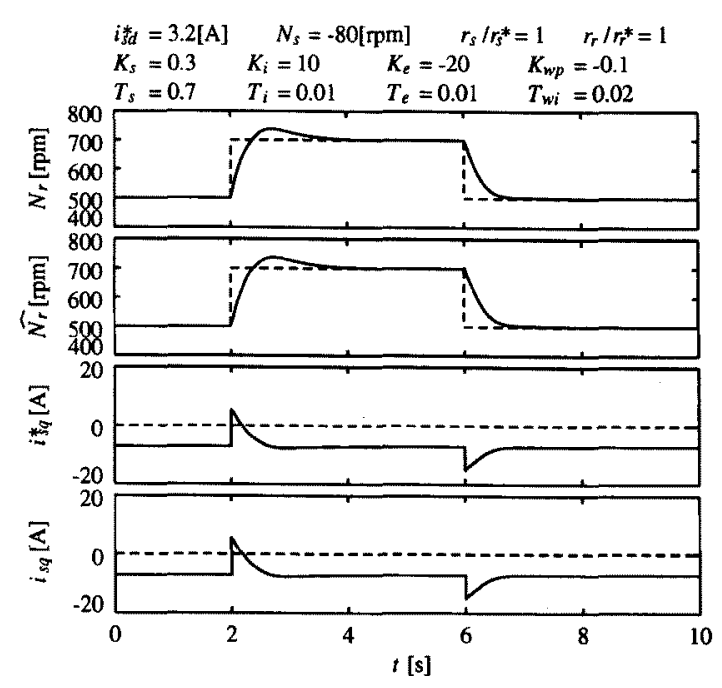

(c) Regenerating mode with $K_{e}=-20$

Fig.5. Transient responses for step change of $N_{r}$ 


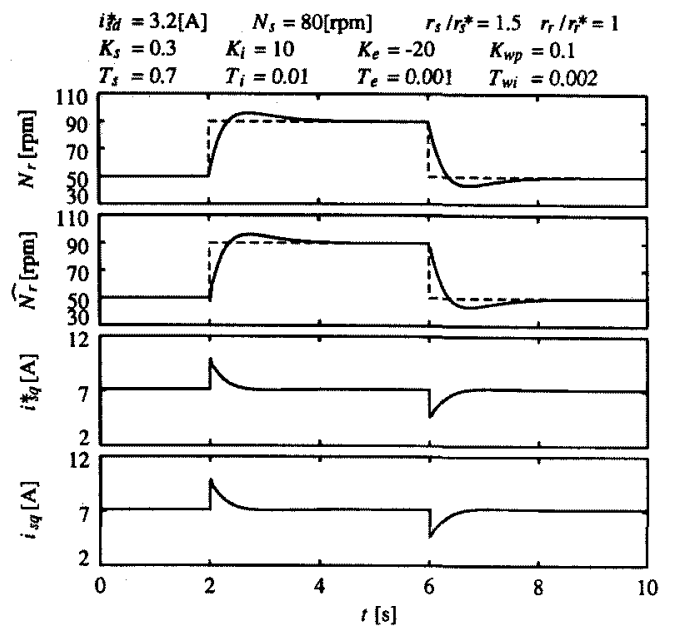

(a) Motoring mode

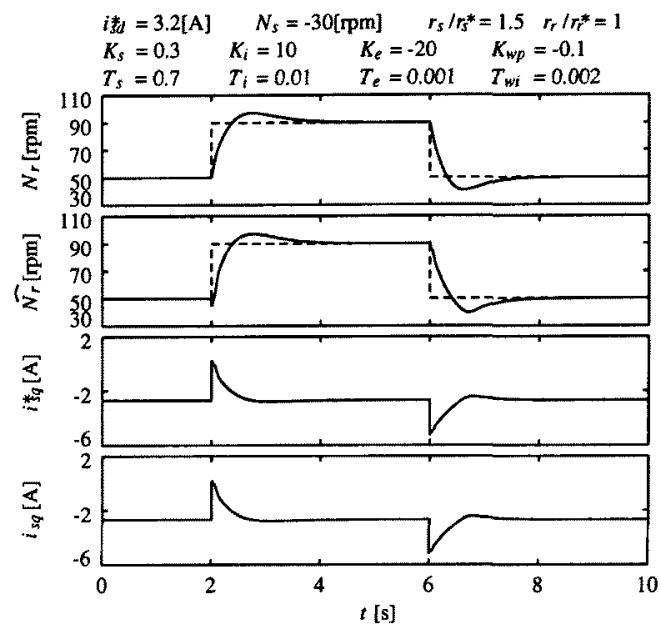

(b) Regenerating mode

Fig.6. Transient responses at lower speed

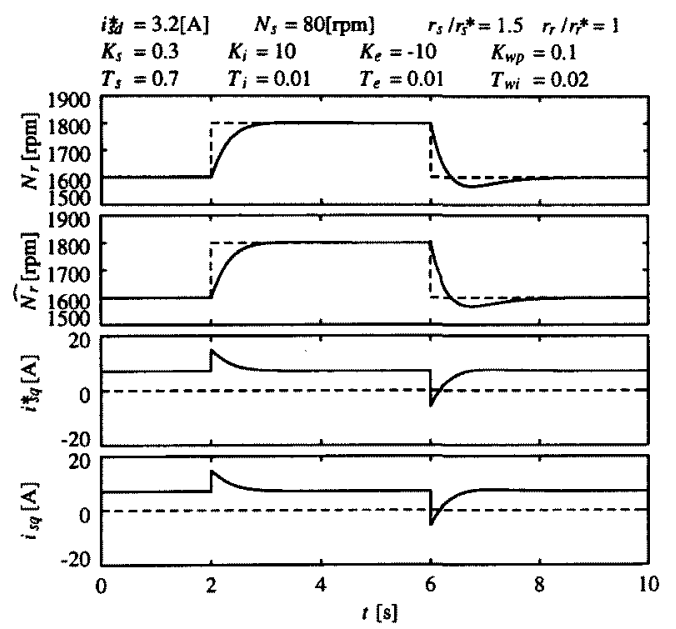

(a) Motoring mode

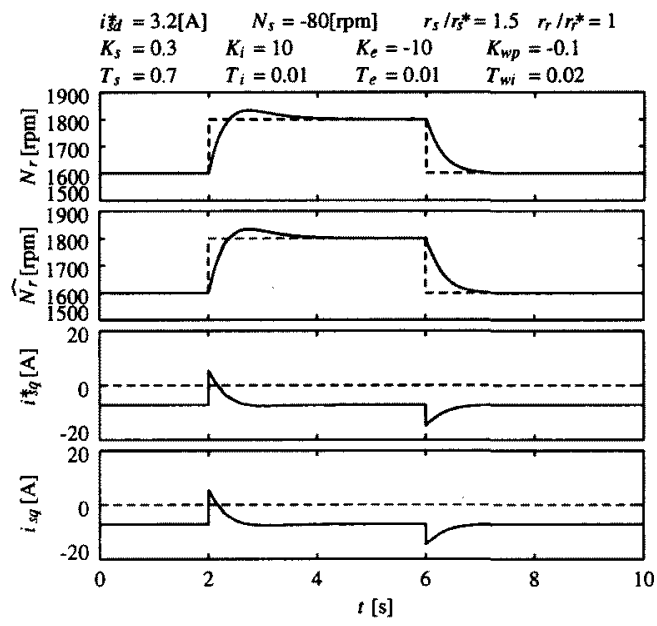

(b) Regenerating mode

Fig.7. Transient responses at higher speed

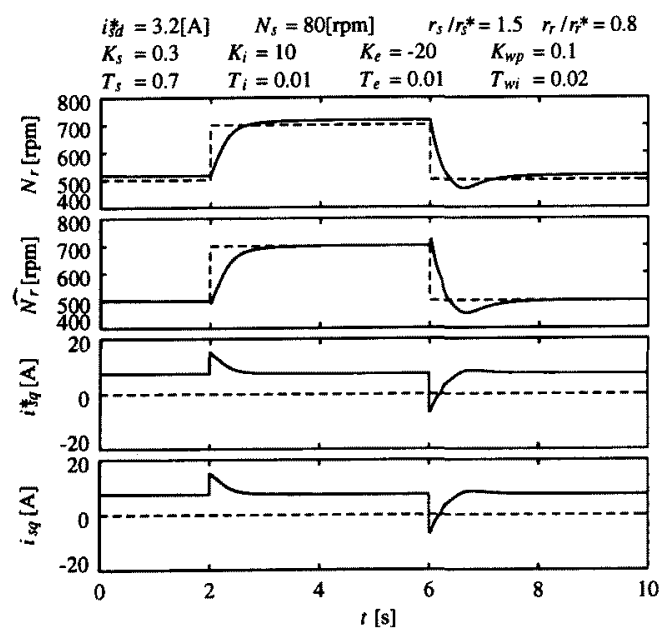

(a) Motoring mode

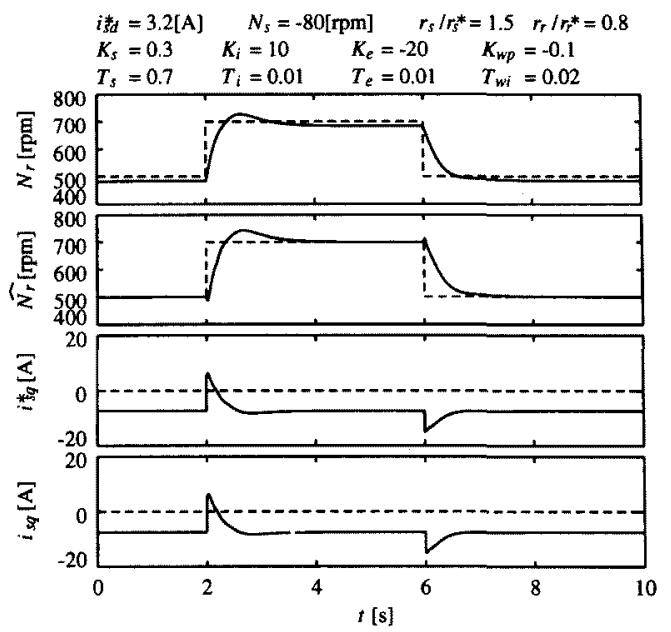

(b) Regenerating mode

Fig.8. Transient responses when $r_{r}=0.8 r_{r o}$ 
work in a wide speed range and is suited to operate in both motoring and regenerating mode.

Fig. 8 shows the result that rotor resistance $r_{r}$ is $0.8 r_{r o}$. Fig.8 (a) and Fig.8 (b) are the results that the motor operates in motoring mode or in regen. erating mode, respectively. From these two fig. ures, we know that the proposed system is stable even if $r_{r}$ is smaller than $r_{r o}$. The speed accuracy of the system is under the influence of rotor resistance variation. The above simulation results confirm the validity of the proposed system and the inherent robustness to stator resistance. In addition, the system gain must be changed depending upon the motor speed, as shown in Table 2 , but it is not very strict. In a practical control system, a hysteresis band of the motor speed can be used to ensure smooth transition between the adjacent gains. Another method that the gains as a smooth function of the motor speed are stored in a lookup table can be also used.

\section{Conclusion}

In this paper, a new method of speed sensorless vector control for induction motors is proposed. The motor speed is estimated by both instantaneous reactive power and variation of magnetizing current. A linear model of the whole system is deduced to analyze system stability. A set of system gain is selected to be suited for operating in the different conditions. Digital simulation shows that the proposed system can work in a much wide speed range and in motoring or regenerating mode without any special technique.

(Manuscript received September 24, 1998, revised January 25,1999 )

\section{References}

(1) C. Schauder, "Adaptive Speed Identification for Vector Control of Induction Motors without Rotational Transducers", IEEE Trans. on Industry Applications, Vol. 28, No. 5, 1992, pp. 1054-1061

(2) T. Chin, "Approaches for Vector Control of Induction Motor without Speed Sensor", Proc. IECON'94, 1994, pp. 1616-1620.

(3) H. Kubota, M. Tsuji, "On-line Tuning of Induction Machine Parameters", Proc. JLASC"95, 1995, S.69S.74.

(4) H. Umida, "The System Configuration of Speed Sensorless Vector Control", Trans. IEE of Japan, Vol. 117-D, N0. 5, 1997,pp. 541-543.
(5) M. Tsuji, E. Yamada, K. Izumi, J. Oyama, "Stability Analysis of a Controlled Current Source-Fed Induction Motor Vector Control System without a Speed Sensor", Trans. IEE of Japan, Vol. 112-D, NO. 9, 1992,pp. 844-851.

(6) F. Z. Peng, T. Fukao, "Robust Speed Identification for Speed-Sensorless Vector Control of Induction Motors", IEEE Trans. on Industry Applications, Vol. 30, No. 5, 1994, pp. 1234-1240.

(7) H. Kubota, K. Matsuse, Y. Hori, "Behavior of Sensorless Induction Motor Drives in Regenerating Mode", Proc. PCC-Nagaoka'97, 1997, pp. 549-552.

(8) M. Tsuji, Y. Ohtsuka, K. Izumi, E. Yamada, "Stability of Speed Sensorless Vector-Controlled Induction Motor using Instantaneous Reactive Power", Proc. JIASC'96, 1996, pp. 319-322.

(9) L. J. Garces, "Parameter Adaption for the SpeedControlled Static AC Drive with a Squirrel-Cage Induction Motor", IEEE Trans. on Industry Applications, Vol. IA-16, No. 2, 1980, pp. 173-178.

\section{Appendix}

$\boldsymbol{A}_{s}=\left[\begin{array}{ccccc}a_{3} & \omega^{*} & a_{1} \sigma_{r} M & a_{1} \omega_{r} M & a_{1} M \psi_{r q} \\ -\omega^{*} & a_{3} & -a_{1} \omega_{r} M & a_{1} \sigma_{r} M & a_{1} M \psi_{r d} \\ \sigma_{r} M & 0 & -\sigma_{r} & \omega^{*}-\omega_{r} & -\psi_{r q} \\ 0 & \sigma_{r} M & \omega_{r}-\omega^{*} & -\sigma_{r} & \psi_{r d} \\ -a_{2} \psi_{r q} & a_{2} \psi_{r d} & a_{2} i_{s q} & -a_{2} i_{s d} & -R_{\omega} / J\end{array}\right]$

$a_{1}=\frac{1}{\sigma L_{s} L_{r}}, a_{2}=\frac{P^{2} M}{4 J L_{r}}, a_{3}=-a_{1}\left(r_{s} L_{r}+\sigma_{r} M^{2}\right)$

$\boldsymbol{B}_{s}=\left[\begin{array}{ccc}\frac{1}{\sigma L_{s}} & 0 & i_{s q} \\ 0 & \frac{1}{\sigma L_{s}} & -i_{s d} \\ 0 & 0 & \psi_{r q} \\ 0 & 0 & -\psi_{r d} \\ 0 & 0 & 0\end{array}\right], \quad \boldsymbol{B}_{L}=\left[\begin{array}{c}0 \\ 0 \\ 0 \\ 0 \\ -\frac{P}{2 J}\end{array}\right]$

$A_{z}=\left[\begin{array}{cccccc}0 & -\frac{a_{4} K_{\omega p}}{T_{\omega i}} & 0 & 0 & \frac{\sigma L_{s} i_{s d}^{*} K_{\omega p}}{T_{q} T_{\omega i}} & \frac{a_{4} K_{\omega p} K_{s}}{T_{\omega i}} \\ 0 & 0 & 0 & 0 & 0 & -\frac{K_{s}}{T_{s}} \\ 0 & 0 & 0 & 0 & 0 & 0 \\ 0 & \frac{K_{p}}{T_{i}} & 0 & 0 & 0 & \frac{K_{s} K_{p}}{T_{i}} \\ 0 & \frac{1}{T_{q}} & 0 & 0 & -\frac{1}{T_{q}} & -\frac{K_{s}}{T_{q}} \\ \frac{1}{T_{\omega}}-\frac{a_{4} K_{\omega p}}{T_{\omega}} \frac{K_{e} T_{i}}{K_{p} T_{e} T_{\omega}} & 0 & \frac{\sigma L_{s} i_{s d}^{*} K_{\omega p}}{T_{q} T_{\omega}} & a_{5}\end{array}\right]$

$a_{4}=e_{s d}^{*}+2 \sigma L_{s} \omega^{*} i_{s q}^{*}+\sigma L_{s} i_{s d}^{*} / T_{q}$

$a_{5}=-1 / T_{\omega}+a_{4} K_{\omega p} K_{s} / T_{\omega}$ 


$$
\begin{aligned}
& \boldsymbol{A}_{x}=\left[\begin{array}{ccccc}
0 & 0 & 0 & 0 & 0 \\
0 & 0 & 0 & 0 & 0 \\
-\frac{K_{p}}{T_{i}} & 0 & 0 & 0 & 0 \\
0 & -\frac{K_{p}}{T_{i}} & 0 & 0 & 0 \\
0 & 0 & 0 & 0 & 0 \\
-\frac{K_{e}}{T_{\omega}} & 0 & 0 & 0 & 0
\end{array}\right] \\
& \boldsymbol{B}_{z}=\left[\begin{array}{ccc}
-\frac{K_{\omega p}}{T_{\omega i}} i_{s q}^{*} & \frac{K_{\omega p}}{T_{\omega i}} i_{s d}^{*} & -\frac{K_{\omega p}}{T_{\omega i}} a_{6} \\
0 & 0 & 0 \\
0 & 0 & 0 \\
0 & 0 & 0 \\
0 & 0 & 0 \\
-\frac{K_{\omega p}}{T_{\omega}} i_{s q}^{*} & \frac{K_{\omega p}}{T_{\omega}} i_{s d}^{*} & -\frac{K_{\omega p}}{T_{\omega}} a_{6}
\end{array}\right] \\
& a_{6}=L_{s} i_{s d}^{* 2}+\sigma L_{s} i_{s q}^{2} \\
& \boldsymbol{B}_{r}=\left[-\frac{a_{4} K_{\omega p} K_{s}}{T_{\omega i}} \frac{K_{s}}{T_{s}} 0 \frac{K_{4} K_{p}}{T_{i}} \frac{K_{s}}{T_{q}}-\frac{a_{4} K_{\omega p} K_{s}}{T_{\omega}}\right]^{T} \\
& \boldsymbol{F}_{x}=\left[\begin{array}{ccccc}
-K_{p} & 0 & 0 & 0 & 0 \\
0 & -K_{p} & 0 & 0 & 0 \\
0 & 0 & 0 & 0 & 0
\end{array}\right], \quad \boldsymbol{F}_{r}=\left[\begin{array}{c}
-a_{7} K_{s} \\
K_{s}\left(K_{p}+\sigma_{r}^{*} L_{s}\right) \\
\sigma_{r}^{*} K_{s} / i_{s d}^{*}
\end{array}\right] \\
& a_{7}=\sigma L_{s}\left(\omega^{*}+\sigma_{r}^{*} i_{s q}^{*} / i_{s d}^{*}\right) \\
& \boldsymbol{F}_{z}=\left[\begin{array}{lllllc}
0 & -a_{7} & 1 & 0 & 0 & a_{7} K_{s}-\sigma L_{s} i_{s q}^{*} \\
0 & K_{p}+\sigma_{r}^{*} L_{s} & 0 & 1 & 0 & L_{s} i_{s d}^{*}-K_{s}\left(K_{p}+\sigma_{r}^{*} L_{s}\right) \\
0 & \sigma_{r}^{*} / i_{s d}^{*} & 0 & 0 & 0 & 1-\sigma_{r}^{*} K_{s} / i_{s d}^{*}
\end{array}\right] \\
& \boldsymbol{C}=\left[\begin{array}{lllllllllll}
0 & 0 & 0 & 0 & 1 & 0 & 0 & 0 & 0 & 0 & 0
\end{array}\right]
\end{aligned}
$$

Mineo Tsuji (Member) He received the D. Eng.

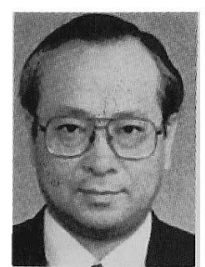
degree from Kyushu University in 1981. He became a lecturer at $\mathrm{Na}$ gasaki University in April 1981 and an associate professor in October 1983. His current interest is Variable Speed Drives and Applications of Control Theory. He is a member of IEEE, the Society of System Control and Information Processing of Japan, and the Japan Society of Applied Electromagnetics and Mechanics. He received the Paper Award from the Institute of Electrical Engineers of Japan in 1998.

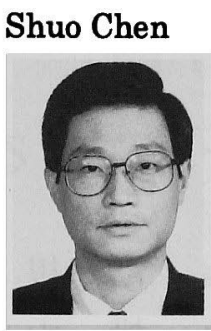

(Student-member) He received the B. S. degree from Nanjing University of Aeronautics and Astronautics in 1985 . He was a visiting researcher at Nagasaki University from April 1993 to March 1994. He became a lecturer at Fuzhou University in 1994 . He is currently a $\mathrm{Ph}$. D. student at Nagasaki University

from April 1997. His principal research is in Variable Speed Drives, Applications of Control Theory and Machatronics. He is a student member of IEEE.

Katsuhiro Izumi (Member) He received the D.

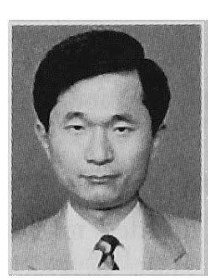
Eng. degree from Kyushu University in 1996. He is currently a research associate in the Department of Electrical and Electronic Engineering at Nagasaki University. His current research is the Power Electronics and Power Control System with processors. He is a member of

IEEE, the Society of Instrumentation and Control Engineers of Japan, and the Japan Society of Applied Electromagnetics and Mechanics. He received the Paper Award from the Institute of Electrical Engineers of Japan in 1998.

Eiji Yamada (Member) He received the D. Eng.

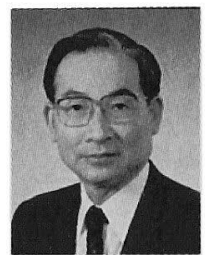

Degree from Kyushu University in 1964, where he was appointed a research associate. He became an associate professor at Nagasaki University in 1968 and a professor in 1974. He has been involved in research on Power Electronics and Control of Electric Apparatus. He is a senior member of IEEE, and a member of the Institute of Electronics Information and Communication Engineers of Japan, the European Power Electronics and Drives Association, the Japan Society of Applied Electromagnetics and Mechanics, and the Society of Instrumentation and Control Engineers of Japan. He received the Paper Award from the Institute of Electrical Engineers of Japan in 1998. 